\title{
A convenient enantioselective decarboxylative aldol reaction to access chiral $\alpha$-hydroxy esters using $\beta$-keto acids
}

\author{
Zhiqiang Duan ${ }^{1}$, Jianlin Han ${ }^{1,2}$, Ping Qian ${ }^{1}$, Zirui Zhang ${ }^{1}$, Yi Wang ${ }^{* 1,3}$ \\ and Yi Pan ${ }^{1,3}$
}

\section{Full Research Paper}

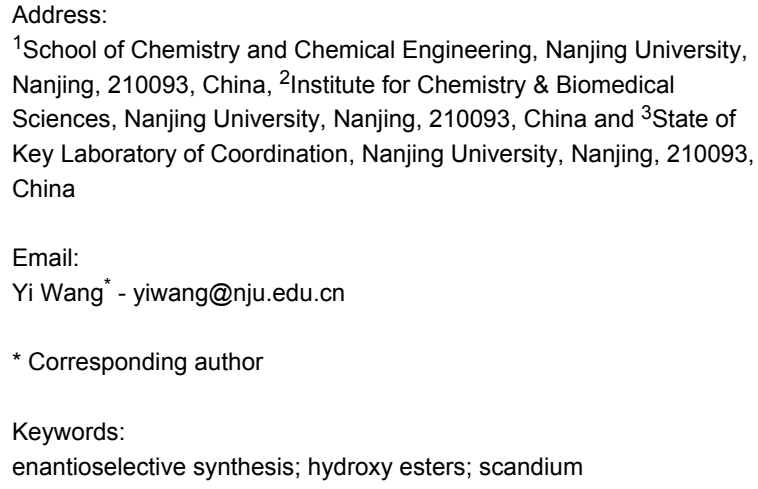

${ }^{1}$ School of Chemistry and Chemical Engineering, Nanjing University, Nanjing, 210093, China, ${ }^{2}$ Institute for Chemistry \& Biomedical Sciences, Nanjing University, Nanjing, 210093, China and ${ }^{3}$ State of Key Laboratory of Coordination, Nanjing University, Nanjing, 210093, China

Email:

Yi Wang* - yiwang@nju.edu.cn

* Corresponding author

Keywords:

enantioselective synthesis; hydroxy esters; scandium

\author{
Beilstein J. Org. Chem. 2014, 10, 969-974. \\ doi:10.3762/bjoc. 10.95 \\ Received: 08 January 2014 \\ Accepted: 10 April 2014 \\ Published: 29 April 2014 \\ Associate Editor: V. M. Dong \\ (C) 2014 Duan et al; licensee Beilstein-Institut. \\ License and terms: see end of document.
}

\begin{abstract}
We show a convenient decarboxylative aldol process using a scandium catalyst and a PYBOX ligand to generate a series of highly functionalized chiral $\alpha$-hydroxy esters. The protocol tolerates a broad range of $\beta$-keto acids with inactivated aromatic and aliphatic $\alpha$-keto esters. The possible mechanism is rationalized.
\end{abstract}

\section{Introduction}

The catalytic enantioselective construction of tertiary carbon centres is a major challenge in organic chemistry. The nucleophilic attack of carbonyls appears as a common procedure, affording chiral tertiary alcohols which are ubiquitous in the biological sciences and pharmaceutical industry [1-6]. The decarboxylative aldol reaction, broadly used for the generation of ester enolate equivalents by the promotion of releasing $\mathrm{CO}_{2}$, has become an appealing method to access chiral tertiary alcohols.
Taking advantage of this rigid reactivity, several unique catalytic decarboxylative aldol transformations of $\beta$-keto acids with various protic aldehydes have been developed [7-10] (Figure 1). High enantioselectivities were achieved with one point-binding aldehydes and two-point binding $\beta$-keto acids under mild reaction conditions. The lack of strong Lewis acids or very basic intermediates enabled it to tolerate functionalities that would normally be incompatible with ester enolates, for 
<smiles>[R]C(=O)CC(=O)O</smiles><smiles>[R]C(=O)CC([R])O</smiles>

$\mathrm{R}^{1}=\mathrm{PhS}, \mathrm{ArO}, \mathrm{EtO}, t-\mathrm{Bu}$, etc.

$\mathrm{R}^{2}=\mathrm{Ph}, \mathrm{Py}$, thiophene, alkyl, etc.

Figure 1: Decarboxylative aldol reactions of $\beta$-keto acids with aldehydes.

instance, hydroxy groups, phenols, enolizable aldehydes and carboxylic acids.

Other less reactive carbonyl derivatives such as isatins [11,12], ketimines [13] and sulfonylimines [14] have also been employed with $\beta$-keto acids in the decarboxylative addition processes.

$\alpha$-Keto esters as surrogates of aldehydes for the generation of chiral alcohols by stereocontrolled nucleophilic alkylation [1519], alkynylation [20,21], 1,2-addition [22-26] and aldol reaction [27-32] have been developed. Various nucleophiles such as organometallics, boronic acids and unsaturated ketones can be tolerated in this context (Figure 2).

We presume that relatively hindered $\alpha$-keto esters could also be engaged as aldehydes in the decarboxylative aldol reactions with $\beta$-keto acids, which would provide a practical and effi- cient route to access $\alpha$-hydroxy esters in an enantioseletive fashion (Figure 3).

\section{Results and Discussion}

By investigating different Lewis acids with various chiral PyBox ligands 4-8 (Table 1), we discovered that $\mathrm{Sc}(\mathrm{OTf})_{3}$ and tridentate PyBox ligand 6a could promote the decarboxylative aldol reaction of $\beta$-keto acid $\mathbf{1 a}$ with $\alpha$-keto ester $\mathbf{2 a}$ in excellent yield with high enantioselectivity in toluene (Table 1, entry 9). Trace amount of side product acetophenone was formed through decarboxylative protonation of $\beta$-keto acid $\mathbf{1 a}$, which was commonly observed in the case of chiral organic base catalysed decarboxylative additions.

Further optimisation of the reaction conditions showed that $\mathrm{CHCl}_{3}$ was the best solvent choice in terms of catalytic activity and asymmetric induction (Table 1 , entry 12 ). Lowering the reaction temperature from $20{ }^{\circ} \mathrm{C}$ to $0{ }^{\circ} \mathrm{C}$ increased the ee<smiles>[R]C(=O)C(=O)OCC</smiles>

$\mathrm{Nu}=\mathrm{Et}_{2} \mathrm{Zn}, \mathrm{Sn}(\text { allyl })_{4}$, alkynes, $\operatorname{ArB}(\mathrm{OH})_{2}$,<smiles>C=C(O[Na])c1ccccc1</smiles>

Figure 2: Nucleophilic reaction of $\alpha$-keto esters to generate tertiary alcohols<smiles>[R]C(=O)CC(=O)OC(=O)C([R])(O)CC([R])=O</smiles> 
Table 1: Evaluation of ligands and optimisation of reaction conditions.<smiles>CCOC(=O)C(=O)c1ccccc1</smiles>

$1 \mathrm{a}$

\author{
ligand 4-8 (12 mol \%) \\ metal salt $(10 \mathrm{~mol} \%)$
}

solvent, rt, $24 \mathrm{~h}$<smiles>CCOC(=O)C(O)(CC(=O)c1ccccc1)c1ccccc1</smiles>

3a<smiles>CC1N=C(c2cccc(C3=N[C@@H](C)C(c4ccccc4)O3)n2)OC1c1ccccc1</smiles><smiles></smiles><smiles>[R]C1COC(c2cccc(C3=NC([R])CO3)n2)=N1</smiles><smiles>CC(C)(C)C1COC(CC2=NC(C(C)(C)C)CO2)=N1</smiles><smiles>c1ccc(C2N=C(CC3=N[C@@H](c4ccccc4)C(c4ccccc4)O3)C(c3ccccc3)O2)cc1</smiles>

\begin{tabular}{|c|c|c|c|c|c|}
\hline Entry & Ligand & Metal salt & Solvent & Yield $(\%)^{b}$ & ee $(\%)^{c}$ \\
\hline 1 & 4 & $\mathrm{Sc}(\mathrm{OTf})_{3}$ & toluene & 93 & 27 \\
\hline 2 & 4 & $\mathrm{Yb}(\mathrm{OTf})_{3}$ & toluene & 90 & 19 \\
\hline 3 & 4 & $\mathrm{La}(\mathrm{OTf})_{3}$ & toluene & 90 & 11 \\
\hline 4 & 4 & $\ln (\mathrm{OTf})_{3}$ & toluene & 88 & 5 \\
\hline 5 & 4 & $\mathrm{Hf}(\mathrm{OTf})_{4}$ & toluene & 91 & 15 \\
\hline 6 & 7 & $\mathrm{Cu}(\mathrm{OTf})_{2}$ & toluene & 85 & 5 \\
\hline 7 & 8 & $\mathrm{Cu}(\mathrm{OTf})_{2}$ & toluene & 83 & 17 \\
\hline 8 & 5 & $\mathrm{Sc}(\mathrm{OTf})_{3}$ & toluene & 91 & 33 \\
\hline 9 & $6 a$ & $\mathrm{Sc}(\mathrm{OTf})_{3}$ & toluene & 95 & 76 \\
\hline 10 & $6 b$ & $\mathrm{Sc}(\mathrm{OTf})_{3}$ & toluene & 94 & 45 \\
\hline 11 & $6 a$ & $\mathrm{Sc}(\mathrm{OTf})_{3}$ & $\mathrm{CH}_{2} \mathrm{Cl}_{2}$ & 90 & 49 \\
\hline 12 & $6 a$ & $\mathrm{Sc}(\mathrm{OTf})_{3}$ & $\mathrm{CHCl}_{3}$ & 93 & 79 \\
\hline 13 & $6 a$ & $\mathrm{Sc}(\mathrm{OTf})_{3}$ & $\mathrm{CH}_{3} \mathrm{CN}$ & 88 & 33 \\
\hline 14 & $6 a$ & $\mathrm{Sc}(\mathrm{OTf})_{3}$ & THF & 89 & 27 \\
\hline 15 & $6 a$ & $\mathrm{Sc}(\mathrm{OTf})_{3}$ & $\mathrm{CHCl}_{3}$ & 91 & $62^{d}$ \\
\hline 16 & $6 a$ & $\mathrm{Sc}(\mathrm{OTf})_{3}$ & $\mathrm{CHCl}_{3}$ & 95 & $84^{e}$ \\
\hline
\end{tabular}

aReaction conditions: 1a $(0.2 \mathrm{mmol}), \mathbf{2 a}(0.1 \mathrm{mmol})$, metal salt $(10 \mathrm{~mol} \%)$, ligand $(12 \mathrm{~mol} \%)$. b ${ }^{\mathrm{l}} \mathrm{solated}$ yield after column chromatography. CDetermined by HPLC analysis using a chiralcel IA column. ${ }^{\mathrm{d}} 10 \mathrm{mg} 4 \AA$ molecular sieves were added. ${ }^{e} \mathrm{At} 0{ }^{\circ} \mathrm{C}$ for $48 \mathrm{~h}$.

value from $79 \%$ to $84 \%$ (Table 1, entry 16). The addition of $4 \AA$ molecular sieves was not able to accelerate the reaction or to improve the enantioselectivity (Table 1, entry 15).

The reaction scope was investigated by using different aryl and alkyl substituted $\beta$-keto acids and $\alpha$-keto esters. Evaluating the results of products $\mathbf{3 b}-\mathbf{f}$, suggested that the $\alpha$-keto esters with electron-withdrawing substituents were more favoured than those with electron-donating groups (Scheme 1, 3b-e). The ortho substituted phenyl $\alpha$-keto ester gave a lower ee (41\%) than those with para substituents ( $49-84 \%$ ee, 3b-e). Aliphatic $\alpha$-keto esters provided the corresponding aldol products with moderate enantioselectivity (56-77\% ee, 3g-i). Also, different $\beta$-keto acids 1a-d with aromatic and alkyl substituents afforded chiral hydoxy esters $\mathbf{3} \mathbf{j}-\mathbf{n}$ in high yields and good enantioselectivities (49-75\%).

We also examined different $\mathrm{R}^{3}$ groups of $\alpha$-keto esters $\mathbf{2 a - d}$. Under the established conditions, an ethyl group afforded the highest yield with the best selectivity. The enantioselectivity did not improve in the cases of methyl, isopropyl or benzyl esters (Table 2, entries 2-4).

The mechanism of the reaction was proposed based on the kinetic studies of the malonic acid half thioester system by Shair [33]. Essentially $\beta$-keto acids can undergo decarboxyla- 


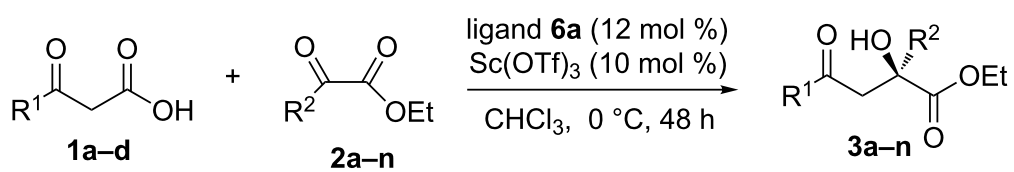<smiles>CCOC(=O)[C@](O)(CC(=O)c1ccccc1)c1ccccc1</smiles>

3a: $90 \%, 84 \%$ ee<smiles>CCOC(=O)[C@](O)(CC(=O)c1ccccc1)c1ccc(F)cc1</smiles>

3b: $90 \%, 78 \%$ ee<smiles>CCOC(=O)[C@](O)(CC(=O)c1ccccc1)c1ccc(Br)cc1</smiles>

3c: $91 \%, 81 \%$ ee<smiles>CCOC(=O)[C@](O)(CC(=O)c1ccccc1)c1ccc(OC(F)(F)F)cc1</smiles>

3d: $92 \%, 76 \%$ ee<smiles>CCOC(=O)[C@@](O)(CC(=O)c1ccccc1)c1ccc(OCC)cc1</smiles><smiles>CCOC(=O)C(O)(CC(=O)c1ccccc1)c1ccccc1F</smiles><smiles>CCOC(=O)[C@](C)(CCc1ccccc1)CC(=O)c1ccccc1</smiles>

3g: $93 \%, 77 \%$ ee<smiles>CCOC(=O)C(C)(O)CC(=O)c1ccccc1</smiles>

3h: $88 \%, 60 \%$ ee<smiles>CCOC(=O)[C@](O)(CC(=O)c1ccccc1)C(C)C</smiles><smiles>CCOC(=O)[C@](O)(CC(=O)c1ccc(F)cc1)c1ccccc1</smiles>

3j: $88 \%, 75 \%$ ee

3k: $91 \%, 60 \%$ ee<smiles>[Mg]</smiles><smiles>CCOC(=O)[C@](O)(CC(=O)c1ccc(I)cc1)c1ccccc1</smiles>

3I: $92 \%, 60 \%$ ee<smiles>CCOC(=O)[C@](O)(CC(=O)c1ccc2ccccc2c1)c1ccccc1</smiles>

3m: $93 \%, 59 \%$ ee<smiles>CCOC(=O)C(O)(CC(=O)CC)c1ccccc1</smiles>

3n: $81 \%, 49 \%$ ee

Scheme 1: Asymmetric decarboxylative aldol reaction of various $\beta$-keto acids with $\alpha$-keto esters under optimised conditions. Reaction conditions: $1(0.2 \mathrm{mmol}), 2(0.1 \mathrm{mmol}), \mathrm{Sc}(\mathrm{OTf})_{3}(10 \mathrm{~mol} \%)$, ligand $6 \mathrm{a}(12 \mathrm{~mol} \%)$. Isolated yield after column chromatography. Enatiomeric excess determined by HPLC analysis using a chiralcel column. ${ }^{\mathrm{a}} 4(\mathrm{~S})$-PyBox $6 \mathrm{a}$ was used.

Table 2: Effect of the ester group on the $\alpha$-keto esters with $\beta$-keto acid 1a. ${ }^{a}$<smiles>O=C([OH2+])CC(=O)c1ccccc1</smiles>

$1 \mathrm{a}$

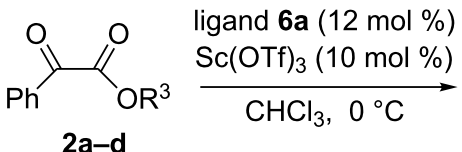

2a-d<smiles>O=C(O)C(=O)CC(O)(c1ccccc1)c1ccccc1</smiles>

3

\begin{tabular}{lllll}
\hline Entry & $\mathrm{R}^{3}$ & Time $(\mathrm{h})$ & ${\text { Yield }(\%)^{\mathrm{b}}}$ & ee $(\%)^{\mathrm{c}}$ \\
\hline 1 & Et, 2a & 48 & 95 & $\mathbf{8 4}, \mathbf{3 a}$ \\
2 & Me, 2b & 36 & 93 & $47, \mathbf{3 0}$ \\
3 & iPr, 2c & 48 & 91 & $71, \mathbf{3 p}$ \\
4 & Bn, 2d & 48 & 89 & $67, \mathbf{3 q}$ \\
\hline
\end{tabular}

aReaction conditions: $1 \mathrm{a}(0.2 \mathrm{mmol})$, a-keto esters 2 ( $0.1 \mathrm{mmol})$, scandium (10 mol \%), and ligand $\mathbf{6 a}(12 \mathrm{~mol} \%)$. ${ }^{\mathrm{b}} / \mathrm{solated}$ yield after column chromatography. ${ }^{\mathrm{C}}$ Determined by HPLC analysis using chiralcel column. 
tion or deprotonation to generate enolates. Though in the case of enzymatic reactions, decarboxylation occurs first to form the enolates, followed by condensation with esters; it is believed that in the scandium-catalysed aldol process of $\beta$-keto acid, similar to the case of malonic acid half thioesters, decarboxylation happens after the addition to the ester (Scheme 2). First, deprotonation and enolisation of 9 followed by addition of $\alpha$-keto ester 2 gives intermediate 11. After decarboxylation to afford 12, a protonation step occurs late in the reaction pathway to form the aldol product $\mathbf{3}$ and completes the mechanistic cycle.

\section{Conclusion}

We have described a new convenient decarboxylative aldol protocol to generate highly functionalised chiral $\alpha$-hydroxy esters employing a $\mathrm{Sc}(\mathrm{OTf})_{3}$ and PyBox catalytic system. A broad range of inactivated $\alpha$-keto esters were proven to be tolerated. The possible mechanism of the reaction was also rationalized. Further investigations to explore the reaction scope are underway.

\section{Supporting Information}

\section{Supporting Information File 1}

Experimental and analytical data.

[http://www.beilstein-journals.org/bjoc/content/

supplementary/1860-5397-10-95-S1.pdf]

\section{Acknowledgements}

We gratefully acknowledge the financial support from the National Natural Science Foundation of China (No. 21102071) and the Fundamental Research Funds for the Central Universities (No. 1107020522 and No. 1082020502). The Jiangsu 333 program (for Pan) and Changzhou Jin-Feng-Huang program (for Han) are also acknowledged.

\section{References}

1. Cuzzupe, A. N.; Di Florio, R.; White, J. M.; Rizzacasa, M. A. Org. Biomol. Chem. 2003, 1, 3572-3577. doi:10.1039/b308028e<smiles>[R]C(=O)C(=O)OCC</smiles> 
2. Bunte, J. O.; Cuzzupe, A. N.; Daly, A. M.; Rizzacasa, M. A. Angew. Chem., Int. Ed. 2006, 45, 6376-6380. doi:10.1002/anie.200602507

3. Nicewicz, D. A.; Satterfield, A. D.; Schmitt, D. C.; Johnson, J. S. J. Am. Chem. Soc. 2008, 130, 17281-17283. doi:10.1021/ja808347q

4. Rogers, E. W.; Molinski, T. F. J. Org. Chem. 2009, 74, 7660-7664. doi:10.1021/j0901007v

5. Hayashi, Y.; Yamaguchi, H.; Toyoshima, M.; Okado, K.; Toyo, T.; Shoji, M. Chem.-Eur. J. 2010, 16, 10150-10159. doi:10.1002/chem.201000795

6. Zhang, F.-M.; Peng, L.; Li, H.; Ma, A.-J.; Peng, J.-B.; Guo, J.-J.; Yang, D.; Hou, S.-H.; Tu, Y.-Q.; Kitching, W. Angew. Chem., Int. Ed. 2012, 51, 10846-10850. doi:10.1002/anie.201203406

7. Lalic, G.; Aloise, A. D.; Shair, M. D. J. Am. Chem. Soc. 2003, 125, 2852-2853. doi:10.1021/ja029452x

8. Magdziak, D.; Lalic, G.; Lee, H. M.; Fortner, K. C.; Aloise, A. D.; Shair, M. D. J. Am. Chem. Soc. 2005, 127, 7284-7285. doi:10.1021/ja051759j

9. Lou, S.; Westbrook, J. A.; Schaus, S. E. J. Am. Chem. Soc. 2004, 126, 11440-11441. doi:10.1021/ja045981k

10. Singjunla, Y.; Baudoux, J.; Rouden, J. Org. Lett. 2013, 15, 5770-5773. doi:10.1021/ol402805f

11. Zhong, F.; Yao, W.; Dou, X.; Lu, Y. Org. Lett. 2012, 14, 4018-4021. doi:10.1021/ol301855w

12. Duan, Z.; Han, J.; Qian, P.; Zhang, Z.; Wang, Y.; Pan, Y. Org. Biomol. Chem. 2013, 11, 6456-6459. doi:10.1039/c3ob41460d

13. Yuan, H.-N.; Wang, S.; Nie, J.; Meng, W.; Yao, Q.; Ma, J.-A. Angew. Chem., Int. Ed. 2013, 52, 3869-3873. doi:10.1002/anie.201210361

14. Jiang, C.; Zhong, F.; Lu, Y. Beilstein J. Org. Chem. 2012, 8, 1279-1283. doi:10.3762/bjoc.8.144

15. DiMauro, E. F.; Kozlowski, M. C. J. Am. Chem. Soc. 2002, 124 , 12668-12669. doi:10.1021/ja026498h

16. DiMauro, E. F.; Kozlowski, M. C. Org. Lett. 2002, 4, 3781-3784. doi:10.1021/ol026315w

17. Wieland, L. C.; Deng, H.; Snapper, M. L.; Hoveyda, A. H. J. Am. Chem. Soc. 2005, 127, 15453-15456. doi:10.1021/ja053259w

18. Zheng, K.; Qin, B.; Liu, X.; Feng, X. J. Org. Chem. 2007, 72, 8478-8483. doi:10.1021/jo701491r

19. Blay, G.; Fernández, I.; Muñoz, M. C.; Pedro, J. R.; Recuenco, A.; Vila, C. J. Org. Chem. 2011, 76, 6286-6294. doi:10.1021/jo2010704

20. Jiang, B.; Chen, Z.; Tang, X. Org. Lett. 2002, 4, 3451-3453. doi:10.1021/ol026544i

21. Infante, R.; Gago, A.; Nieto, J.; Andrés, C. Adv. Synth. Catal. 2012, 354, 2797-2804. doi:10.1002/adsc.201200185

22. Ganci, G. R.; Chisholm, J. D. Tetrahedron Lett. 2007, 48, 8266-8269. doi:10.1016/j.tetlet.2007.09.137

23. Duan, H.-F.; Xie, J.-H.; Qiao, X.-C.; Wang, L.-X.; Zhou, Q.-L. Angew. Chem., Int. Ed. 2008, 47, 4351-4353. doi:10.1002/anie.200800423

24. Crespo-Peña, A.; Monge, D.; Martín-Zamora, E.; Álvarez, E.; Fernández, R.; Lassaletta, J. M. J. Am. Chem. Soc. 2012, 134, 12912-12915. doi:10.1021/ja305209w

25. Wang, H.; Zhu, T.-S.; Xu, M.-H. Org. Biomol. Chem. 2012, 10, 9158-9164. doi:10.1039/c2ob26316e

26. Zhu, T.-S.; Jin, S.-S.; Xu, M.-H. Angew. Chem., Int. Ed. 2012, 51, 780-783. doi:10.1002/anie.201106972

27. Akullian, L. C.; Snapper, M. L.; Hoveyda, A. H. J. Am. Chem. Soc. 2006, 128, 6532-6533. doi:10.1021/ja061166o
28. Ogawa, S.; Shibata, N.; Inagaki, J.; Nakamura, S.; Toru, T.; Shiro, M. Angew. Chem., Int. Ed. 2007, 46, 8666-8669. doi:10.1002/anie.200703317

29. Frings, M.; Atodiresei, I.; Runsink, J.; Raabe, G.; Bolm, C. Chem.-Eur. J. 2009, 15, 1566-1569. doi:10.1002/chem.200802359

30. Luo, J.; Wang, H.; Han, X.; Xu, L.-W.; Kwiatkowski, J.; Huang, K.-W.; Lu, Y. Angew. Chem., Int. Ed. 2011, 50, 1861-1864. doi:10.1002/anie.201006316

31. Moteki, S. A.; Han, J.; Arimitsu, S.; Akakura, M.; Nakayama, K.; Maruoka, K. Angew. Chem., Int. Ed. 2012, 51, 1187-1190. doi:10.1002/anie.201107239

32. Bastida, D.; Liu, Y.; Tian, X.; Escudero-Adán, E.; Melchiorre, P. Org. Lett. 2013, 15, 220-223. doi:10.1021/ol303312p

33. Fortner, K. C.; Shair, M. D. J. Am. Chem. Soc. 2007, 129, 1032-1033. doi:10.1021/ja0673682

\section{License and Terms}

This is an Open Access article under the terms of the Creative Commons Attribution License

(http://creativecommons.org/licenses/by/2.0), which permits unrestricted use, distribution, and reproduction in any medium, provided the original work is properly cited.

The license is subject to the Beilstein Journal of Organic Chemistry terms and conditions:

(http://www.beilstein-journals.org/bjoc)

The definitive version of this article is the electronic one which can be found at: doi:10.3762/bjoc. 10.95 